\title{
Kurukh Language
}

National Cancer Institute

\section{Source}

National Cancer Institute. Kurukh Language. NCI Thesaurus. Code C153989.

A Dravidian language spoken by the Oraon and Kisan tribespeople of India, Bangladesh, Nepal, and Bhutan. 